\title{
Une nouvelle approche de la grammaire
}

\author{
Jean-Paul Narcy-Combes
}

\section{OpenEdition}

\section{Journals}

Édition électronique

URL : http://journals.openedition.org/asp/4299

DOI : 10.4000/asp.4299

ISSN : 2108-6354

\section{Éditeur}

Groupe d'étude et de recherche en anglais de spécialité

\section{Édition imprimée}

Date de publication : 1 octobre 1993

Pagination : 259-272

ISSN : 1246-8185

\section{Référence électronique}

Jean-Paul Narcy-Combes, « Une nouvelle approche de la grammaire », ASp [En ligne], 2 | 1993, mis en ligne le 03 mars 2014, consulté le 05 mai 2019. URL : http://journals.openedition.org/asp/4299 ; DOI : $10.4000 / a s p .4299$

Ce document a été généré automatiquement le 5 mai 2019.

Tous droits réservés 


\title{
Une nouvelle approche de la grammaire
}

\author{
Jean-Paul Narcy-Combes
}

\section{Avant-propos}

1 Que les collègues qui ont assisté à cette communication veuillent bien excuser le fait que le texte présenté ci-dessous présente quelques différences dans sa forme avec ce qu'ils ont entendu. Des contraintes de temps, une date de remise des manuscrits justifiée, mais très proche, ne m'ont pas permis de reprendre la transcription de la cassette pour la rendre acceptable à la lecture. Afin de respecter les délais, je préfère proposer le texte d'une conférence faite au colloque du LEND à Montécatini en octobre 1992, sous le titre : «Errare humanum est, Les erreurs, faut-il prévenir pour guérir?», et souligner dans l'avant-propos les différences. Je sollicite également l'indulgence des organisateurs de ce colloque dont les actes seront publiés sous forme de livre en Italie.

2 La conférence de Montécatini insistait particulièrement sur les causes d'erreur et leur traitement, la communication de Grenoble sur les conséquences de ces phénomènes sur la mise en place d'une approche nouvelle de la grammaire. À Montécatini, les différentes étapes de l'approche grammaticale ont été définies, mais elles n'ont pas été décrites, un atelier a permis aux volontaires de le faire par la suite. À Grenoble, ces étapes ont été décrites, elles n'apparaîtront qu'en annexe ici.

3 L'esprit des deux communications reste le même ; toute approche naturelle a ses limites, l'apprenant a besoin d'aide pour que la structuration interne s'effectue de manière satisfaisante, cette aide s'appelle la grammaire, elle l'aidera à dominer les risques d'erreur (et les erreurs révéleront l'état de sa grammaire interne à un instant $\mathrm{T}$ ). Ce retour à la grammaire ne devrait pas s'accompagner d'un retour à d'anciennes pratiques, au contraire.

4 Il s'agira de créer une gestion nouvelle des concepts qui conduira à remettre en cause aussi bien le métalangage, que la présentation de la plupart des grammaires d'aujourd'hui qui privilégient la forme au détriment du sens, qui favorisent plus la compréhension 
(relation formes-sens) que l'expression (relation sens-forme) et qui de ce fait n'aident pas les apprenants à effectuer les opérations mentales qu'exigent les échanges spontanés.

Le praticien qui s'interroge sur l'apprentissage d'une langue seconde (L2) en aura une image différente suivant l'usage qu'il fera du kaléidoscope que composent les diverses théories qui décrivent les phénomènes qui le conditionnent. Néanmoins, négliger une seule de ces théories risquerait de donner une vision incomplète d'une réalité complexe. Cette communication sur l'erreur est le résultat d'une réflexion théorique menée de pair avec des expérimentations pratiques. Son but est de justifier le besoin d'une sensibilisation aux erreurs dans le cadre d'un auto-apprentissage guidé et de présenter un point de vue différent, mais complémentaire de ce que présente Second Language Research de juin 1991.

6 Trois grands points seront abordés successivement : une réflexion théorique, les causes d'erreur et leur traitement, et les moyens de sensibiliser l'apprenant.

\section{Réflexion théorique}

7 Comme le rappelle D. Larsen Freeman (Scarcella et Long), tout apprentissage se résume par le schéma 1.

Schéma 1

\begin{tabular}{|lcr|}
\hline & la boite nolre & \\
INPUT & INTAKE & OUTPUT \\
Relation forme $\rightarrow$ sens & & Relation sens $\rightarrow$ forme \\
\cline { 2 - 3 } & &
\end{tabular}

8 Le passage de la compréhension d'une relation forme - sens à une possibilité d'exprimer soi-même une relation sens - forme requiert une structuration interne des données accompagnée de leur mémorisation que les chercheurs appellent «intake». C'est sans doute dans le renversement de la relation forme - sens vers une relation sens - forme qu'il faut chercher la source des difficultés des apprenants.

En dix ans, la théorie a évolué de la notion "d'input compréhensible » (Krashen), à celle "d'output compréhensible» (Swain ou Scarcella \& Long), puis à la notion «d'input négocié » (Long, in Scarcella \& Long). La justification de cette évolution se trouve partiellement dans les phénomènes de « nativisation » (dans MacLaughlin \& Long, Larsen Freeman) et dans la nécessité d'avoir des processus ascendants impeccables pour traiter efficacement l'« output compréhensible » (Rod Ellis).

Définissons ces deux phénomènes :

- La nativisation (théorie de l'acculturation, cf. MacLaughlin, et Larsen Freeman, Long) est la façon dont l'apprenant intègre la L2 selon des critères internes de sa L1, ou de la grammaire universelle. Son « intake » ne correspondra pas nécessairement à l'« input » de ce fait.

- Les processus ascendants (bottom up) sont les processus mentaux qui permettent de remonter des formes au sens, par opposition aux processus descendants (top down) qui eux permettent d'aller de la connaissance du monde et du contexte au sens par anticipation et compensation.

11 Participer à un échange requiert le jeu en parallèle de ces deux types de processus. La compréhension peut s'effectuer avec un minimum de travail ascendant; en revanche, la production exigera un assez gros travail au bas niveau. Tout «input» devrait être 
décomposé en ses composantes sémantiques et ses composantes linguistiques. L'« intake » ne sera utile au plan linguistique que si l'« input linguistique » a été saisi, c'est ce que voulait dire Ellis quand il écrivait que «l'input compréhensible ne peut être efficace que si les processus ascendants sont très efficaces ». Cette efficacité sera difficilement atteinte en situation naturelle ou en début d'apprentissage, c'est ce qui justifie la notion d'«input » compréhensible négocié.

Dans un échange "naturel ", la difficulté de comprendre oblige l'apprenant à entamer une procédure de négociation du sens, qui, en ralentissant le débit et réduisant temporairement la charge sémantique, permet à l'apprenant de se concentrer sur l'« input» linguistique (ainsi sera évité un début de nativisation). De plus, parler «force l'apprenant à prêter attention aux moyens d'expression » (Swain, cité par Ellis) et donc permet de mettre en place la relation sens-forme.

Nous avons ainsi compris le passage de l'input compréhensible à « l'input négocié » dans lequel apparaît en fait la notion «d'output compréhensible » (c'est celui qui permet de négocier l'input). Les apprentissages guidés mettent cette "négociation de l'input » en œuvre sous forme d'approche grammaticale progressive; un apprentissage en autoformation risque de laisser l'apprenant plus libre sur ce point et favorisera peut-être plus la nativisation.

D'un autre côté, un apprentissage plus traditionnel, risque de pécher en se focalisant trop sur les processus de bas niveau (lexique, gestion de la morphosyntaxe), sans prendre conscience que c'est à un niveau plus élevé (conception, planification, contrôle) qu'il faudrait agir (Gaonach'). Que l'on soit donc dans une situation d'auto-apprentissage guidé ou d'apprentissage guidé, une attention particulière devra être portée à la structuration interne, et donc aux risques d'erreur et à leur traitement, ce qui nous conduit à notre deuxième point.

\section{Les causes d'erreurs et leur traitement}

Le modèle d'apprentissage suivant (Narcy 1990) est une tentative de prise en compte des difficultés de la structuration interne, il intègre la difficulté d'obtenir une structuration interne immédiate et le fait que cette dernière ne peut être mesurée que par une production langagière.

Schéma 2

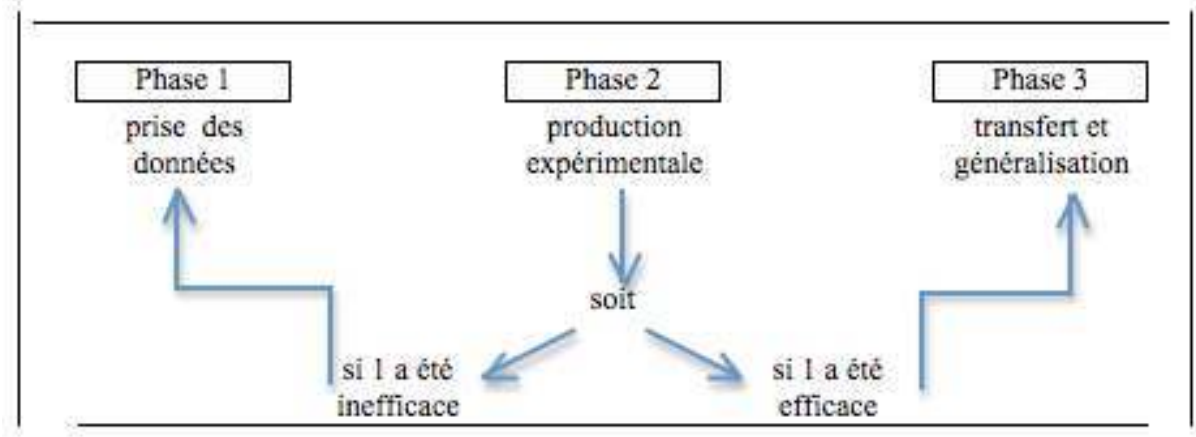

En effet s'il y a inattention à la forme par surcharge cognitive (Gaonach' ou Swain), jeu des processus descendants, ou analyse incorrecte de la forme (nativisation) l'intake ne 
sera pas «correct » et cela se traduira par un « output » non correct, qui sera le signe qu'un travail sur « l'input » devra être reproposé à l'apprenant.

Pour que la charge cognitive soit réduite en production, il faut que les procédures de traitement des données soient automatisées et efficaces (cette forme de connaissance qui permet le traitement et l'emploi du savoir s'appelle « connaissance procédurale »). Il faut donc que des pré-requis procéduraux soient en place : entraînement aux automatismes (faire le choix voulu) et automatismes pertinents (par opposition au conditionnement béhavioriste, il faut savoir faire le bon choix en fonction de la situation et de son intention de jouer sur cette situation).

Pour que l'analyse de la forme soit correcte, il faut, au préalable, que les concepts véhiculés par cette forme soient clairement identifiés par l'apprenant : cela exige des prérequis conceptuels.

i, pour un Francophone qui apprend l'anglais, les concepts de quantités continue et discontinue, par exemple, ne sont pas clairement maitrisés. Certains apprenants parviennent à les maîtriser grâce à de l'« input » en L2 uniquement, d'autres auront sans doute besoin d'un travail spécifique de mise en place de ces concepts (il y a beaucoup de fromages en France, par opposition à il y a beaucoup de fromage dans son assiette). De plus, pour que l'ensemble fonctionne il faut également que l'on n'ait pas préjugé des aptitudes de l'apprenant (cf. Skehan), en particulier ici de sa capacité d'« analyse linguistique ».

Il est clair qu'en début d'apprentissage, ces pré-requis ne seront pas en place et l'apprenant aura besoin d'aide: au plan conceptuel, partout où la L2 présente des différences à ce niveau avec la $\mathrm{L} 1$; au plan procédural, là où il faudra automatiser la relation intention - conception (analyse conceptuelle), recherche des formes pour éviter des courts circuits conception/forme nativisée.

21 Un exemple de ces courts circuits serait l'expression de la durée en anglais. Pour des francophones, il y a souvent : intention d'exprimer une durée, lien automatique durée/il y $a$, association il y a/there is, expression d'une durée en anglais par emploi de there is.

Ce qui manque alors c'est l'analyse conceptuelle selon les critères de L2, une simple correction par répétition ne saurait agir sur ce manque, puisqu'il n'y aurait pas travail de haut niveau.

\section{Il faudra donc éviter les corrections où l'on donne la forme correcte et où} l'apprenant n'a qu'à la répéter.

Rappelons que la mise en place du conditionnement skinnérien se faisait dans des exercices dits structuraux où l'apprenant manipulait des formes sans passer par les phases suivantes :

- intention de communiquer

- conception de l'énoncé

- choix de la relation sens-forme adéquate, c'est-à-dire que le travail de « haut niveau » était escamoté.

Ce qui précède conduit à entraîner l'apprenant à percevoir les formes qui lui poseront problème, à percevoir avec quelles formes elles s'opposent et quel est son risque d'erreur (travail sur l'« input ", puis à l'entraîner à faire des choix pertinents dans des situations contextualisées où on essaiera de limiter la charge cognitive [travail sur l'« output »]).

Si le travail sur l'« input » a été malmené, on obtiendra un premier type d'erreur causé par un savoir (dit déclaratif) inexact. 
Si le travail sur l'« output» a été inefficace, lorsqu'il y aura surcharge cognitive ou focalisation sur le sens, il y aura un second type d'erreur, causé par des procédures inefficaces.

\section{On peut se demander si des erreurs dont les causes sont si différentes doivent être traitées de la même façon.}

Carroll et Swain (1992) nous rappellent que ce qu'ils nomment "negative feedback», la correction des erreurs, fonctionne mieux avec des apprenants avancés. Ils nous montrent, par ailleurs, que la correction des erreurs ne permet pas la généralisation des règles. De leurs recherches et des nôtres, nous sommes tentés de suggérer que la correction des erreurs doit s'appliquer au deuxième type d'erreurs (erreurs procédurales) plutôt qu'au premier [erreurs déclaratives].

Il reste à savoir quand, comment, par qui ces erreurs seront corrigées (cf. Chaudron, qui se demande lesquelles de ces erreurs seront corrigibles)? En effet, Ellis nous indique que la correction des erreurs la plus efficace porte sur :

- les morphème simples : par exemple le $s$ du pluriel en anglais ou une erreur du type je vais à manger que font les Italiens en français.

- <\#ITALIQUES\#></\#ITALIQUES\#>les cas où la relation forme/fonction est transparente: par exemple la relation passé/présent en anglais est plus transparente que la relation général/ particulier (marquée par les formes simple et continue). De même des erreurs sur l'emploi de this/that seront reprises plus efficacement par correction que des erreurs sur l'emploi de l'article the.

Ellis nous rappelle également que cette correction conduit parfois à une surproduction de certaines formes. Il nous indique aussi quel genre d'apprenant est le plus susceptible de bénéficier de ce type de correction, en citant Johnson (1988).

Cet apprenant aura : le désir de corriger ses erreurs, une représentation mentale correcte de la bonne forme (pas de nativisation), la perception qu'une erreur a été commise et la possibilité d'être en situation de communication authentique.

En résumé, le traitement des erreurs imposera deux stratégies suivant l'étape de l'apprentissage où se situera l'apprenant :

Avant un seuil qui reste à définir, il faudra jouer sur l'« input » en apportant une aide à la déconstruction, et en vérifiant l'efficacité de ce travail par de l'« output compréhensible » sans surcharge cognitive (cf. plus haut).

Des retours constants à la phase 1 permettront de consolider la connaissance déclarative en mettant en place des règles de fonctionnement efficaces (automatismes). On peut penser qu'une analyse du fonctionnement de la relation forme-sens évitera la nativisation. Reste à définir la forme que prendra cette analyse, et à savoir s'il est possible d'aller au-delà de ce qu'Ellis appelle une «sensibilisation » à la règle. La question se pose de mesurer si on peut totalement contrecarrer les effets de la nativisation.

Après un seuil qui reste à définir : il faudra jouer essentiellement sur l'« output », mais en situation de production naturelle, pour éviter la fossilisation des erreurs procédurales. Comment reste un problème?

À l'Université de Compiègne, nous avons mis en place un système d'indication d'erreurs en production spontanée pour apprenants avancés. Un locuteur natif anime des petits groupes de quatre apprenants, il dispose d'un clavier sur lequel chaque touche représente un des concepts les plus malmenés par des francophones en anglais. Quand une erreur est 
commise, la touche voulue est activée et elle allume le nom du concept (durée, aspect, etc.) sur un panneau lumineux. Dans la plupart des cas l'apprenant reformule son énoncé sans aide, la forme correcte était donc connue déclarativement. Il arrive même que le simple mouvement de la main induise une correction spontanée. Une expérience menée sur quinze heures et quinze semaines avec un groupe pilote et un groupe témoin a montré que les apprenants du groupe pilote faisaient sensiblement plus de progrès au niveau de la structuration des énoncés que ceux du groupe témoin. Cette expérience avait essayé de respecter les conditions de succès décrites par Johnson. Dans un contexte non pénalisant, elle a été fort bien vécue par les apprenants. Néanmoins, elle n'a pas conduit à la disparition totale des erreurs.

Le praticien doit donc se rappeler que là encore il s'agira de sensibilisation: rendre l'apprenant conscient des points où son « savoir déclaratif s'est mal procéduralisé ». Ce qui justifie cette sensibilisation est le fait que, sans elle, l'apprenant risque fort de voir ses erreurs se «fossiliser», ce qui rendrait tout effort de se corriger quasiment impossible dès lors. L'apprenant doit donc être prévenu à temps.

L'erreur est humaine, il faut néanmoins la prévenir: avant un seuil, par des tâches appropriées, et après un seuil, par une correction appropriée.

\section{Comment sensibiliser l'apprenant à ces phénomènes ? (apprendre à apprendre)}

De ce qui précède, nous pouvons suggérer le schéma méthodologique suivant.

Avant le seuil

Avant le seuil, on pourrait suggérer une approche heuristique (par la découverte), qui proposerait aux apprenants successivement des tâches de découverte du lien forme/sens ou des liens formes/sens qui s'opposent, d'analyse conceptuelle, pour s'assurer que les concepts sont bien perçus, de manipulation décontextualisée (automatismes primaires), de manipulation contextualisée avec choix pertinents (ou non s'il n'y en a pas pour une structure/forme donnée) et complexification progressive des contextes (automatismes « intelligents » traités par les processus de haut niveau).

Ainsi serait mise en place la relation sens/forme, et on éviterait peut-être plus facilement les phénomènes de nativisation. Il est tout à fait possible de concevoir ce travail dans le cadre d'un apprentissage en autonomie. Les besoins des apprenants sont en général très différents, même au niveau de la grammaire, et il est tout à fait concevable de créer des séquences de travail où en fonction de ses besoins un apprenant pourrait aborder divers concepts, à son rythme, dans un centre de ressources (voir en annexe, des exemples de telles tâches).

Après le seuil

Après le seuil, tâches de production libre : avec indication conceptuelle des erreurs, pour mettre en place l'auto-contrôle par une sensibilisation à quelles erreurs l'apprenant comment dans ce cas, alors qu'il connait la bonne forme (exemple d'indication conceptuelle, si un apprenant dit en anglais, ${ }^{*}$ I am going in Paris tomorrow, pointer le doigt en avant pour indiquer une erreur sur la direction, afin que l'apprenant se reprenne sans autre aide, voir également la machine à indiquer les erreurs décrites dans cet article).

Ou bien sans feedback immédiat, mais avec auto-contrôle et/ou feedback différé. 
Cela implique bien sûr un guidage méthodologique avant le seuil, et une sensibilisation après le seuil. Un travail en autonomie totale sera moins efficace sur ce point qu'un travail en autonomie guidée. Ce travail de sensibilisation diffère quelque peu de celui qui est décrit par Michael Sharwood Smith, en grande partie parce que cette sensibilisation se fait dans le cadre d'apprentissages auto-guidés, heuristiques et conceptuels.

\section{Conclusion}

Tout comme un apprentissage sur le tas ne sera efficace que si l'apprenant est un très bon apprenant de langue, un auto-apprentissage ne sera vraiment efficace que si l'apprenant dispose de tâches qui le sensibilisent aux relations sens-forme et forme-sens en L2 et lui évitent les pièges que lui tendent la nativisation, le fonctionnement des processus descendants, etc., et ensuite d'un guidage qui lui montre les faiblesses de la procéduralisation de son savoir.

De nombreux paramètres restent inconnus, en particulier le point où se situe le «seuil » qui, une fois franchi, permet de passer d'une stratégie à une autre (travail sur l'« input » $\neq$ indication des erreurs).

Comme nous l'avons vu, les tâches sur l'«input » peuvent être conçues pour un travail individuel autonome. Rien ne dit que les exercices de grammaire traditionnels suffiront, loin de là. Un grand effort de créativité reste donc à faire pour fournir aux centres d'autoapprentissage les exercices voulus qui compléteront l'ensemble des tâches plus directement communicatives proposées aux apprenants. Il serait, bien sûr, inquiétant de privilégier le travail sur la langue au détriment des objectifs communicatifs. Il resterait donc à définir plus finement les parts respectives de ce travail sur la langue et du travail plus globalement communicatif dans un apprentissage donné.

Rappelons néanmoins que toute sensibilisation aux erreurs restera conditionnée par les paramètres suivants :

- progression en $\mathrm{U}$, cf. Rod Ellis (à une amélioration quantitative de la production risque de correspondre une légère déperdition qualitative, voire un " plateau »);

- conditions pour l'apprenant que nous avons déjà citées ;

- variabilité de l'interlangue, cf. Rod Ellis (suivant les contextes, l'apprenant se focalise plus ou moins sur les formes);

- besoin de consolider constamment les acquis (comme le montre l'expérience de Lydia White), donc admettre les régressions inévitables.

51 Il ne s'agirait pas en effet de démotiver les apprenants par des objectifs irréalistes. Si l'erreur est humaine, y prêter trop attention pourrait être diabolique...

\section{BIBLIOGRAPHIE}

Carroll, S., M. Swain et Y. Robergé. 1992. «The role of feedback in adult second language acquisition: Error correction and morphological generalizations ». Applied Linguistics 13, 173-198. 
Chaudron, C. 1988. Second Language Classrooms. Cambridge : Cambridge University Press.

Ellis, R. 1990. Instructed Second Language Acquisition. Oxford : Blackwell.

Gaonach', D. (dir.). 1990. « Les stratégies attentionnelles dans l'utilisation d'une langue seconde ». Le Français dans le Monde, Numéro spécial. fév-mars.

Gass, S. et L. Madden (dir.). 1985. Input in Second Language Acquisition. Cambridge : MA : Newbury House.

Gass, S. et al. (dir.). 1989. Variations in Second Language Acquisition. (2 volumes). Philadelphie : Multilingual Matters.

Gass, S. et J. Schachter. 1989. Perspectives on Second Language Acquisition. Cambridge : Cambridge University Press.

Harley, B. et al. 1990. The Development of Second Language Proficiency. Cambridge : Cambridge University Press.

Larsen-Freeman, D. et M. H. Long. 1991. An Introduction to Second Language Research. Londres : Longman.

MacLaughlin, B. 1987. Theories of Second Language Acquisition. Londres : Edward Arnold.

Narcy, J.-P. 1990. Apprendre une langue étrangère. Paris : Éditions d'Organisation.

Narcy, J.-P. 1991. « La prise de conscience des problèmes linguistiques conduit-elle à une réduction des erreurs commises en production libre? ». Actes du Colloque Acquisition des langues secondes, Grenoble 3, mai 1991.

Pienemann, M. 1992. Teachability Theory. Sydney : The University of Sydney, Language Acquisition Research Centre.

Sharwood Smith, M. 1991. "Speaking to many minds: on the relevance of different types of language information for the L 2 learner ». Second Language Research 7/2, 118-132.

Skehan, P. 1989. Individual Differences in Second Language Acquisition. Londres : E. Arnold.

White, L. 1991. «Adverb placement in second language acquisition: some effects of positive and negative evidence in the classroom ». Second Language Research 7/2, 133-161.

\section{ANNEXES}

\section{Annexe 1}




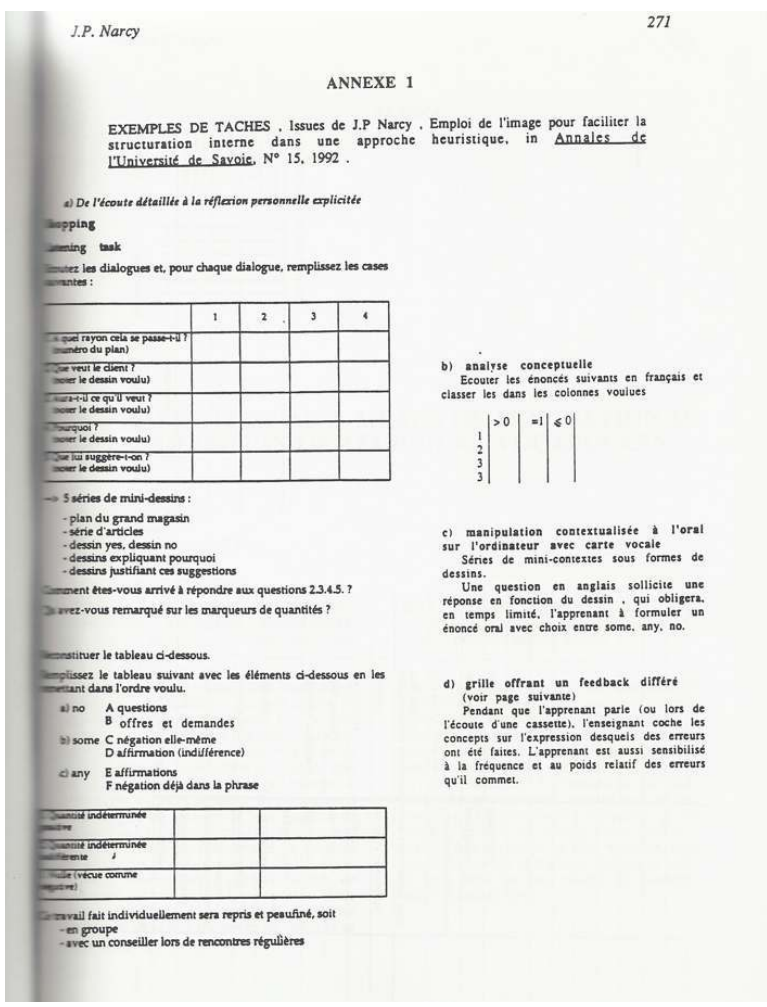

\section{Annexe 2}
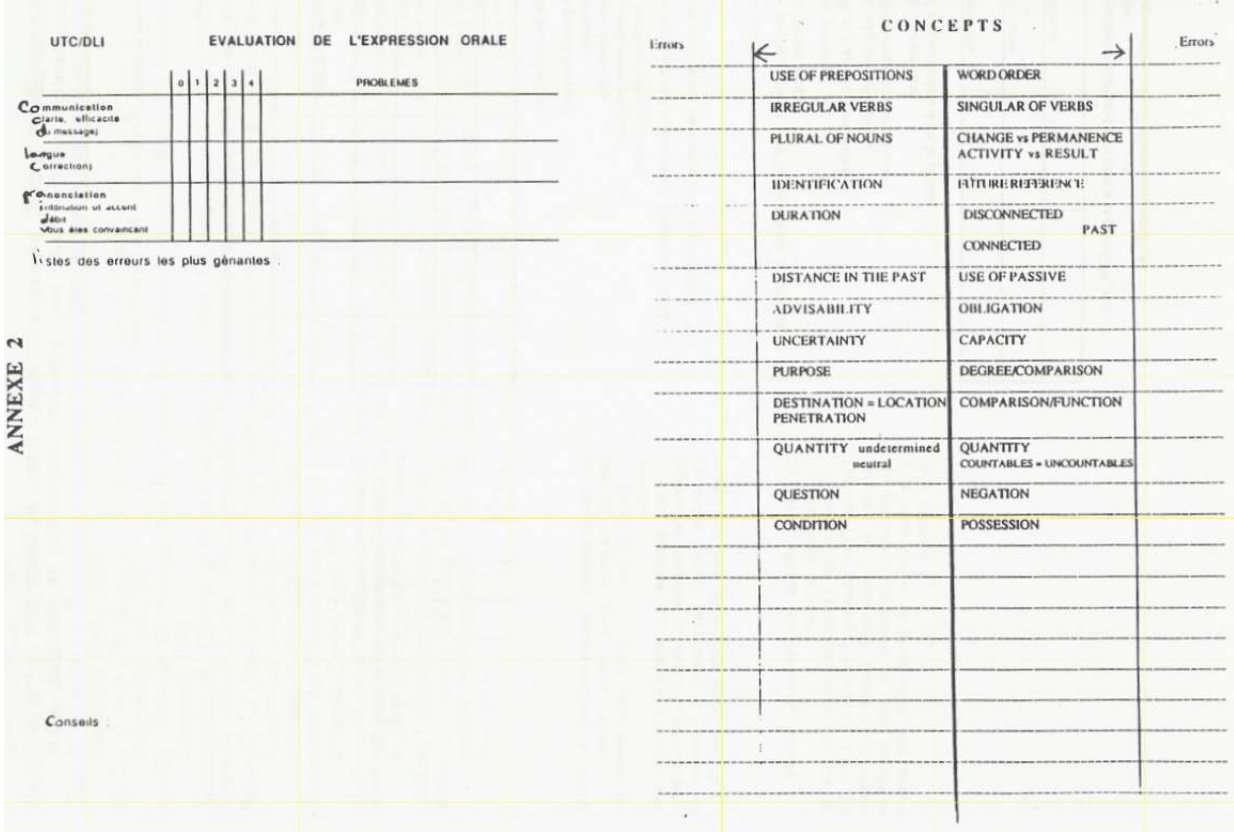

\section{RÉSUMÉS}

Une approche nouvelle de la grammaire est imposée par la prise en compte des processus mentaux qui conditionnent l'apprentissage et les échanges langagiers. Cette prise en compte 
induit des changements au niveau des priorités et des tâches qu'il convient de proposer aux apprenants. Il faudra se rappeler que le retour à la grammaire devra s'accompagner d'une réflexion approfondie sur la hiérarchisation des objectifs linguistiques et des objectifs communicationnels afin de ne pas relancer le balancier trop loin.

A new approach to grammar teaching is imposed by a better knowledge of the mental processes that accompany the learning and the use of an L2. This induces changes at the level of priorities and at the level of the tasks that learners should perform. However the teacher will have to remember that going back to grammar requires a better selective analysis of the priorities between linguistic and communicative objectives so as not to swing the pendulum too far back.

\section{INDEX}

Mots-clés : grammaire, linguistique, processus mental

Keywords : grammar, linguistics, mental process

\section{AUTEUR}

\section{JEAN-PAUL NARCY-COMBES}

IUFM de Picardie et COSTECH, Université de Compiègne. jean-paul.narcy-combes@wanadoo.fr 